\title{
COVID 19 Y DIABETES MELLITUS TIPO 1 EN LA INFANCIA. CUBA. INFORME PRELIMINAR
}

\author{
Covid 19 and Diabetes Mellitus type 1 in childhood. \\ Cuba. Preliminary report
}

\section{Francisco Carvajal Martínez ${ }^{\mathrm{a}}$, Julieta García Saez ${ }^{\mathrm{b}}$, Orlando Araujo Herrerac, José R. Hernández Gómez ${ }^{\mathrm{d}}$, Juliette Navarrete Cabrera ${ }^{e}$, Ivette Pereira Venéreo', Katiuska Martín Duráng, Daymara Espino González ${ }^{\mathrm{h}}$, Jany Casanovas Figueroa', Mónica Carvajal Aballej y Alejandro Ramos Robledok}

Recibido: 26 de marzo, 2021 • Aprobado: 24 de julio, 2021

Cómo citar: Carvajal Martínez F, García Saez J, Araujo Herrera O, Hernández Gómez JR, Navarrete Cabrera J, Pereira Venéreo I, Martín Durán K, Espino González D, Casanovas Figueroa J, Carvajal Aballe M, Ramos Robledo A. Covid 19 y Diabetes Mellitus tipo 1 en la infancia. Cuba. Informe preliminar. cysa [Internet]. [citado 22 de febrero de 2022];6(1):25-33. Disponible en: https://revistas. intec.edu.do/index.php/cisa/article/view/2408

\section{Resumen}

Introducción: en diciembre de 2019 se inició una epidemia por coronavirus SARS-CoV-2, causante de un síndrome de dificultad respiratoria que, posteriormente, se denomina enfermedad COVID-19 y que es probable afecta menos a los niños que a los adultos. En Cuba existen aproximadamente 15,246 contagiados menores de 19 años. La diabetes mellitus tipo 1 (DM1) asociada al Covid-19 puede representar peligro para la vida del paciente.

Objetivo: determinar algunas características de la COVID-19 en la población pediátrica estudiada con diabetes mellitus

\footnotetext{
a Profesor Titular y Consultante de Endocrinología. Especialista de $1^{\text {er }}$ y $2^{\text {do }}$ Grado en Endocrinología, Departamento de Endocrinología Pediátrica. Instituto Nacional de Endocrinología (INEN). La Habana. Cuba. ORCID: 0000-0002-8480-1360, Correo-e: endoped@infomed.sld.cu

${ }^{\mathrm{b}}$ Profesor Auxiliar. Especialista de $1^{\text {er }}$ y $2^{\text {do }}$ Grado en Endocrinología. Hospital Pediátrico Universitario "José Luis Miranda”. Villa Clara. Cuba. ORCID: 0000-002-2192-4574

c Profesor Auxiliar, Especialista de $1^{\text {er }}$ y $2^{\text {do }}$ Grado en Endocrinología. Servicio de Endocrinología Pediátrica. Hospital Pediátrico Docente de Centro Habana. Ciudad la Habana. ORCID: 0000-0001-8972-4512

${ }^{\text {d }}$ Profesor Auxiliar. Especialista de $1^{\text {er }}$ y $2^{\text {do }}$ Grado en Endocrinología, Cuba. ORCID: 0000-0002-9079-9893

e Profesora Auxiliar. Especialista de $1^{\text {er }}$ Grado en Endocrinología y de Medicina General Integral. Instituto de Endocrinología. La Habana. Cuba. ORCID: 0000-0002-6647-882X
}

\begin{abstract}
Introduction: On December 2019, an epidemic due to the SARS-CoV-2 coronavirus began, causing a respiratory distress syndrome, later it is called the COVID-19 disease, it probably affects children less than adults. In Cuba, there are approximately 15,246 infected persons under 19 years of age. Type 1 diabetes mellitus (DM1) associated with Covid-19 can be life-threatening.

Objective: To determine some characteristics of COVID-19 in the pediatric population studied with type 1 diabetes

\footnotetext{
${ }^{\mathrm{f}}$ Profesora Asistente. Especialista de $1^{\text {er }}$ Grado en Medicina General Integral, Especialista de Primer Grado en Endocrinología, Hospital Pediátrico del Cerro, Ciudad la Habana. ORCID: 0000-0003-3371-8855

${ }^{g}$ Profesora Asistente. Especialista de $1^{\text {er }}$ Grado en Medicina General Integral, Especialista de Primer Grado en Endocrinología. Hospital Pediátrico del Cerro, Ciudad la Habana. ORCID: 0000-0003-2165-7530.

${ }^{\text {h }}$ Profesor Asistente. Especialista de $1^{\text {er }}$ Grado en Endocrinología. Servicio de Endocrinología Pediátrica. Hospital Pediátrico Docente de Centro Habana. Ciudad la Habana. ORCID: 0000-0003-2375-4043.

i Profesora Instructor. Especialista de $1^{\text {er }}$ Grado en Endocrinología. Servicio de Endocrinología. Hospital Pediátrico Docente Ciego de Ávila, Morón, Cuba. ORCID: 0000-0002-8763-9821

${ }^{j}$ Especialista de $1^{\text {er }}$ Grado en Medicina General Integral y de $1^{\text {er }}$ Grado de Oncología Clínica, Máster en Enfermedades Infecciosas. Cuba. ORCID: 0000-0002-1453-0526

k Interno Vertical de Endocrinología. Facultad Miguel Enríquez. Instituto Nacional de Endocrinología. Cuba. ORCID: 0000-0003-2239-6520
} 
tipo 1 durante el primer ańo de inicio de la pandemia en Cuba.

Método: se seleccionaron al azar los servicios de endocrinología pediátrica de las provincias: Ciego de Avila, Villa Clara, Pinar del Río y en La Habana (Hospitales Pediátricos Cerro y Centro Habana). Se solicitó cuántos pacientes durante l primer año de la pandemin (marzo 2020-mas durt) is. Estos datos se compararon con el ańo 2019. Se investigó cuanto niños DM1 ingresaron con Covid 19

Resultados: existió incremento (57.3\%) de los pacientes con debut de DM1 durante el primer ańo de la pandemia en comparación con 2019. La presencia de cetosis/cetoacidosis aumentó (58.2\%). Se detectó cuatro pacientes con Covid 19 y DM1, (frecuencia baja: 0, 53 \%).

Conclusiones: el incremento de la DM1 y de cetosis/ cetoacidosis pudiera estar relacionado indirectamente con la pandemia por Covid 19, ya sea por stress, confinamiento, alimentación inadecuada o la combinación de estos factores, es prudente tener un seguimiento a mediano y largo plazo de estos pacientes.

Palabras claves: Covid-19; SARS-CoV-2; diabetes mellitus tipo 1 ; infancia, cetoacidosis.

\section{Introducción}

En diciembre de 2019 se inició una epidemia con un nuevo coronavirus, que fue llamado SARS-CoV-2, causante de un síndrome de dificultad respiratoria. La Organización Mundial de la Salud declaró "Emergencia Internacional de Salud Pública" el 30 de enero del 2020, y el 11 de febrero fue oficializada la enfermedad con el nombre de COVID-19 (coronavirus disease, por su sigla en inglés), y considerada oficialmente como pandemia el 11 de marzo del mismo año. Los coronavirus (CoV) se encuentran muy distribuidos en la naturaleza, tienen la propiedad de infectar a los seres humanos, mamíferos y aves, situación que les permite desarrollar enfermedades. Entre ellas se destacan las pande- mellitus during the first year of the onset of the pandemic in Cuba.

Method: The pediatric endocrinology services of the provinces: Ciego de Ávila, Villa Clara, Pinar del Río and Havana (Hospital Pediátricos Cerro and Centro Habana) were randomly selected. It was requested how many patients during the first year of the pandemic (March arch 2021) debuted with DM1, how many in ketosis / ketoacidosis. These data were compared with 2019. It was investigated how many T1D children were admitted with Covid 19.

Results: There was an increase (57.3\%) in patients with T1D debut during the first year of the pandemic compared to 2019. The presence of ketosis / ketoacidosis increased $(58.2 \%) .4$ patients with Covid 19 and DM1 were detected (low frequency: $0.53 \%$ ).

Conclusions: The increase in DM1 and ketosis/ketoacidosis could be indirectly related to the Covid 19 pandemic, whether due to stress, confinement, inadequate nutrition medium and long-term follow-up of these patients.

Keywords: Covid-19; SARS-CoV-2; type 1 diabetes mellitus; childhood, ketoacidosis

mias anteriores que ocasionaron el síndrome respiratorio agudo (SARS-CoV) en 2003 y el síndrome del virus respiratorio del Oriente Medio (MERS-CoV) en el año 2012. ${ }^{1}$ Hasta el momento, existen a nivel mundial 190 países y 30 territorios con casos de COVID-19. Ascienden alrededor de 166,513,629 los casos confirmados, con 15 millones 766 mil 486 casos activos y 3 millones 455 mil 172 fallecidos. En la región de las Américas se reportan 66,785,695 casos confirmados, el 40,14 \% del total de casos reportados en el mundo, con 8,437,490 casos activos y $1,629,413$ fallecidos.

El virus SARS-CoV-2 probablemente afecta menos a los niños que a los adultos, tanto en la frecuencia como en la severidad ${ }^{1}$. Posiblemente, solo entre
1 y $5 \%$ de pacientes afectados por COVID-19 pertenece al rango de edad pediátrico. En Cuba, el primer reporte de COVID-19 se realizó el 11 de marzo del 2020, por la confirmación de tres pacientes extranjeros ${ }^{1}$, hasta la actualidad, se acumulan alrededor 131,832 casos positivos y 863 fallecidos, y existen un total de 15,246 contagiados con la Covid-19 en edad pediátrica (inferior a 19 ańos) $)^{2}$. Se han efectuado algunas publicaciones sobre Covid-19 $9^{1,3-5}$, sin embargo, esta investigación es la primera que se realiza en un grupo de la población pediátrica cubana sobre Covid-19 y DM1. Además de las afectaciones propias originadas por el Covid-19, numerosas comorbilidades pueden ocasionar peligro para la vida del paciente (cardiopatías, enfermedades cerebrovasculares, hipertensión arterial, afecciones pulmonares, obesidad, etc.). Dentro de estas, ocupa un lugar importante la diabetes mellitus, fundamentalmente la tipo 2 (DM2), que es más frecuente en los grupos poblacionales de mayor edad. No obstante, se enfatiza que la infección por el SARS-CoV-2 en poblaciones con Diabetes Mellitus tipo 1 (DM1) se ha incrementado, favoreciendo la descompensación metabólica, el virus además facilita el debut de esta condición médica. ${ }^{6-20}$

Inmunología de la infección por SARS-CoV-2 en la diabetes mellitus

Los coronavirus son virus de ARN monocatenario de sentido positivo, con un gran genoma de 27 a 32 kilobase empaquetado dentro de una cápside formada por la proteína nucleocápside $(\mathrm{N})$. Una envoltura rodea esta estructura helicoidal y está asociada con tres proteínas estructurales: la proteína de membrana (M) y la proteína de la envoltura (E), que están ambas involucradas en el ensamblaje del virus, y la proteína de punta (S), que media la entrada a las células huésped ${ }^{21-23}$. La proteína $S$ se caracteriza además por una subunidad $S 1$ del dominio de unión al receptor (RBD) que facilita la unión al receptor de la enzima convertidora de angiotensina 2 (ACE2) del huésped para el SARSCoV-2, y una subunidad S2 que es responsable de la fusión de la membrana. ${ }^{24,25}$. El RBD se adhiere a las células huésped a través de la dipeptidilpeptidasa 4 (DPP4) en lugar de ACE2 [5]. Los factores de la célula huésped median aún más la entrada viral a través de dos proteasas de serina, que activan la proteína $S$ para la fusión de la membrana a través de la escisión y ayudan en el procesamiento viral, respectivamente ${ }^{23-26}$

Una vez que se ha producido la infección por SARS-CoV-2, se produce una respuesta compleja y orquestada del sistema inmunitario innato y adaptativo que se correlaciona clínicamente con tres fases propuestas: una fase de viremia, una fase aguda y una fase de recuperación ${ }^{27}$. Los patrones moleculares asociados por receptores de reconocimiento de patrones (es decir, receptores tipo Toll), dan como resultado la expresión de interferones de tipo I y factores inflamatorios que potencian los mecanismos de defensa de los macrófagos y las células asesinas naturales. Las células dendríticas son fagocitos de tejido que combinan la respuesta inmune innata y adaptativa activando los linfocitos $\mathrm{T}$ y los linfocitos $\mathrm{B}$ a través de la presentación de antígenos ${ }^{21}$. El sistema inmunológico adaptativo es esencial para el control de la fase persistente de la infección e implica la producción de anticuerpos monoclonales neutralizantes de las glicoproteínas de la envoltura viral por las células T CD4 + y la destrucción de las células infectadas por virus por las células T CD8 + citotóxicas $^{21}$. En la fase aguda de la infección, el SARS-CoV y el SARS-CoV-2 invaden los linfocitos $\mathrm{T}$ CD4 + y CD8 + dando lugar a apoptosis y linfocitopenia, una señal que se asocia con resultados graves $^{24,27}$

Las células beta infectadas por el SARS-CoV-2 pueden reducir la secreción de insulina. Al mismo tiempo, la tormenta de citocinas que a veces acompaña a la infección por SARS-CoV-2 puede estimular o agravar la resistencia a la insulina. Ambas condiciones pueden conducir a la aparición de la 
hiperglucemia, que a su vez puede empeorar aún más la resistencia a la insulina. Además, la hiperglucemia, a través de la glicosilación no enzimática del receptor de la enzima convertidora de angiotensina 2 (ACE2), puede favorecer aún más la penetración de las células del SARSCoV-2, empeorando la COVID-19. La hiperglucemia, por lo tanto, puede inducir disfunción endotelial y generación de trombos, lo que conduce al daño multiorgánico característico de COVID-19. ${ }^{23-27}$

\section{Objetivos}

General: determinar algunas características de la COVID-19 en la población pediátrica estudiada con diabetes mellitus tipo 1 durante el primer año de inicio de la pandemia en Cuba.

Especificos: 1) Precisar la frecuencia de COVID-19 en un grupo de la población pediátrica cubana con diabetes mellitus tipo 1 (de recién diagnóstico o conocidos), durante el primer ańo de inicio de la pandemia de COVID-19. 2) Comparar la frecuencia de diabetes mellitus tipo 1 en un grupo de la población pediátrica cubana de recién diagnóstico (debut) durante el primer año de inicio de la pandemia de COVID-19 y el año previo (2019). 3) Determinar el número de episodios de cetoacidosis/cetosis diabética en un grupo de la población pediátrica cubana durante el primer año de inicio de la pandemia de COVID-19 y el año previo

\section{Material y método}

Se seleccionó al azar los servicios de endocrinología pediátrica de las siguientes provincias: 1 . Zona Oriental - Ciego de Ávila, 2. Central - Villa Clara, 3. Zona Occidental - Pinar del Río. Además, por la importancia de la capital (La Habana) se evaluó lo informado por los servicios de Endocrinología Pediátrica de los Hospitales Pediátricos Docentes del Cerro y Centro Habana. Se solicitó a cada servicio de Endocrinología pediátrica los datos de los DM1 menores de 19 años de edad: 1- cuántos pacientes durante el primer ańo de la pandemia (marzo 2020-marzo 2021) fueron diagnosticados (debut) con DM1, 2- cuántos con DM1 (debut o conocidos) lo hicieron en cetosis/cetoacidosis y 3- cuántos con diagnóstico de DM1 tipo1 ingresaron con Covid. Además se les solicitó: 4- el total de pacientes con DM1 que atendían en sus provinrecogidos en el año anterior de la pandemia (2019). Se aceptó como debut todo paciente que inició su enfermedad; se aceptó que existía cetoacidosis y cetosis de acuerdo con los criterios establecidos en las Normas Nacionales de Diagnóstico y Tratamiento en Endocrinología.

\section{Resultados}

A. Los pacientes con DM1 que atienden los diferentes servicios hospitalarios son: 1 . Pinar de Río: - 121, 2. Cerro: 123, 3. Centro Habana: 164, 4. Villa Clara: 222 y 5 . Ciego de Ávila: 115. Total: 745 niños.

B. Niños con DM1 y Covid-19: 4, en el Hospital Pediátrico del Cerro 3 (un varón con 15 años y 2 hembras con 11 y 17 ańos de edad, respectivamente), en la provincia de Pinar del Río 1 paciente con Covid-19 y DM1, de 1 ańo de edad (contacto padre y madre positivos), los 4 pacientes ingresaron asintomáticos, con buena evolución. Todos los niños diabéticos que presentaron Covid-19, fue en el transcurso de su enfermedad ninguno como debut, y se dieron de alta favorable, existió bajo porciento de niños con DM1 y Covid-19 (0,53\%). Como se observa en a tabla 1 , se evaluaron en el 2019 a 67 pacientes (V: 30; H: 37) que debutaron con DM1 en los diferentes centros estudiados. Existió incremento en el 1er ańo de la pandemia 90 niños (V: 42; $\mathrm{H}$ : 48). Iqualmente, al analizar los pacientes con cetosis/cetoacidosis se detectó en el año previo (2019) 48 pacientes (V: 24; H: 24), mientras durante el primer año de la pandemia 67 niños (V: 28; H: 39).

Tabla 1. Pandemia COVID-19 y DM1. Cuba. Periodo de tiempo

\begin{tabular}{|c|c|c|c|c|}
\hline \multirow[b]{2}{*}{ Zonas del país } & \multicolumn{2}{|c|}{ AÑ̃ PRE (año 2019) } & \multicolumn{2}{|c|}{ 1er ańo (marzo 2020-marzo 2021) } \\
\hline & debut & cetoacidosis/cetosis & debut & cetoacidosis/cetosis \\
\hline \multicolumn{5}{|l|}{ A- Occidental } \\
\hline (*) Pinar del Río & $\begin{array}{c}15 \\
\text { (V: 5; H: 10) }\end{array}$ & $\begin{array}{c}7 \\
\text { (V: } 3 ; \mathrm{H}: 4)\end{array}$ & $\begin{array}{c}16 \\
\text { (V: } 5 ; \mathrm{H}: 11)\end{array}$ & $\begin{array}{c}6 \\
\text { (V: } 3 ; \mathrm{H}: 3)\end{array}$ \\
\hline \multicolumn{5}{|l|}{$(*)$ Habana (Capital) } \\
\hline (**) Hosp. Ped & 5 & 10 & 4 & 7 \\
\hline Cerro & (V: 2; H 3) & (V: 6; H 4) & (V: $3 ; \mathrm{H}: 1)$ & $(\mathrm{V}: 3 ; \mathrm{H}: 7)$ \\
\hline $\begin{array}{l}(* *) \text { Hosp. Ped } \\
\text { Centro Habana }\end{array}$ & $\begin{array}{c}17 \\
(\mathrm{~V}: 10 ; \mathrm{H}: 7)\end{array}$ & $\begin{array}{c}5 \\
\text { (V: } 2 ; \mathrm{H}: 3)\end{array}$ & $\begin{array}{c}19 \\
\text { (V: } 11 ; \mathrm{H}: 8)\end{array}$ & $\begin{array}{c}10 \\
\text { (V: } 6 ; \mathrm{H}: 4)\end{array}$ \\
\hline \multicolumn{5}{|l|}{ B- Central } \\
\hline$\left.{ }^{*}\right)$ Villa Clara & 22 & 19 & 25 & 19 \\
\hline $\begin{array}{l}(* *) \text { Hosp. Ped } \\
\text { Villa Clara }\end{array}$ & (V: 10; H: 12) & (V: 10; H: 9) & (V: 12; H: 13) & (V: 8; H: 11) \\
\hline \multicolumn{5}{|l|}{ C- Oriental } \\
\hline (*) Ciego de Ávila & 7 & 8 & 26 & 22 \\
\hline $\begin{array}{l}\left({ }^{*}\right) \text { Hosp. Ped. } \\
\text { Ciego de Âvila }\end{array}$ & (V: 3; H: 5) & (V: 3; H: 4) & (V: 11; H: 15) & (V: 8; H: 14) \\
\hline TOTAL: (N) & 67 & 48 & 90 & 67 \\
\hline (\%) & 42.6 & 41.7 & 57.3 & 58.2 \\
\hline Sexo: (V; H) & (V: 30; H: 37) & (V: 24; H: 24) & (V: 42; H48) & (V: 28; H: 39) \\
\hline
\end{tabular}

\section{Discusión}

Se ha señalado baja incidencia del COVID-19 en pacientes pediátricos, diferentes investigadores comentan que en cohortes de 44672 y 75465 casos de enfermos confirmados, solo encuentran 2,1 y $2,4 \%$, respectivamente, de positividad (pacientes pediátricos) $)^{1 .}$ Sin embargo, López et al. ${ }^{5}$ plantean un mayor porcentaje en los menores de 19 años infectados por SARS-CoV-2 (11.3\%), lo que pudiera estar relacionado, según estos autores, con la mayor eficacia de los sistemas de vigilancia establecidos en el país. En este estudio se encontró frecuencia baja $(0.53 \%)$ de nińos DM1 con COVID-19. La causa por lo cual la mayoría de los casos con la COVID-19 en pediatría tienen síntomas menos graves que los adultos no está bien definida, probablemente está relacionada tanto con la exposición a la enfermedad como con los factores del huésped, por ejemplo: los niños por lo general se encuentran bien atendidos en casa y pueden tener relativamente menos oportunidades de exponerse a patógenos o pacientes enfermos; el número de receptores de ACE2 (enzima convertidor de angiotensina 2) es inferior al número apreciado en adultos; el sistema inmunitario en la edad infantil todavía se está desarrollando y puede responder a los patógenos de manera diferente; no son frecuente comorbilidades como hipertensión, enfermedad cardiovascular y diabetes, entre otras. ${ }^{1}$

El impacto del COVID-19 se ha centrado en los pacientes con DM2 adultos, alrededor del 10-55 \% de los pacientes hospitalizados con COVID-19 la padecen, sin embargo, Chao et al., ${ }^{11}$ en una primera publicación de esta situación, señalaron, al estudiar en niños la relación DM2 y COVID-19, que era más 
frecuente los casos al debut durante la pandemia $(82$ pacientes) comparados con el año 2018 (44 niños). Igualmente, al evaluar la frecuencia de cetoacidosis se constató que en el 2018-2019 era menor al $10 \%$ sin embargo, durante el 2020 incrementó al $20 \%$ (incluyendo 2 casos de presentación severa). Por otro lado, Kusuma et al. ${ }^{9}$ señalan gran evidencia de la relación del COVID-19 con la diabetes mellitus tipo 2 (DM2), no obstante, plantean que puede provocar en los diabéticos tipo1 (DM1) descompensación metabólica severa (cetoacidosis y coma hiperosmolar hiperglucémico), así como mayor frecuencia de debut, igualmente que existe incremento de la mortalidad en DM1, principalmente en grupos de mayor edad y con comorbilidades importantes asociadas (enfermedad renal o cardíaca, etc.) Al evaluar la relación COVID-19 con el debut de DM1, Zubkiewicz-Kucharska et al. ${ }^{6}$ encuentran igual incidencia que en ańos anteriores de la pandemia, no obstante, seńalan que las condiciones clínicas eran peores al momento del diagnóstico que en ańos previos. Otros autores constatan incremento del diagnóstico de la DM1 durante la pandemia y enfatizan que existe durante esta etapa aumento del descontrol metabólico (cetoacidosis), siendo su presentación más severa.

En esta investigación, se observó incremento $(57.3 \%)$ de los pacientes al debut (N: 90) de la DM1 durante el primer ańo de la pandemia, comparados con los que iniciaron la enfermedad diabética (N: 67) en el año previo (2019), lo cual está de acuerdo con otros trabajos internacionales que encuentran iguales resultados. También hemos encontrado al evaluar según el sexo, que existió al incremento al debut (comparando el ańo 2019 vs. ler año de la pandemia), año 2019, varones: 30 y hembras: 37; durante la pandemia varones: 42 y hembras: 48. Al evaluar la presencia de cetoacidosis/ cetosis constatamos que (al comparar el año 2019 vs. 1 .er año de la pandemia) existió aumento en ambos sexos (año 2019, varones: 24 y hembras: 24; durante la pandemia varones: 28 y hembras: 39). Debido a que solo 4 nińos con DM1 han tenido COVID-19 $(0.53 \%)$, se considera que los resultados pueden estar relacionados de forma indirecta con la pandemia por COVID-19, ya sea por stress (individual, social o ambos), confinamiento, alimentación familiar inadecuada, poca movilidad de la población, etc. (o varios de estos aspectos juntos). Trevisani et al. ${ }^{8}$ alertan que en una etapa inicial los síntomas del COVID-19 pueden retrasar el diagnóstico de la descompensación metabólica en el DM1, por su parte, Lawrence et al. ${ }^{14}$ opinan que por la necesidad del confinamiento (por el COVID-19) puede enlentecer el momento de la asistencia a instituciones de salud y favorecer el retraso de un diagnóstico oportuno. Por su parte, Sánchez ${ }^{20}$ considera que el control metabólico debido al confinamiento puede mejorar, también se ha insistido que los pacientes con DM y COVID-19 pueden ser más vulnerables a la depresión, ansiedad y otras situaciones estresantes ${ }^{7}$.

En este estudio no se informó sobre ningún fallecido durante la pandemia en DM1 menores de 19 años. Consideramos que estos resultados pueden ser debido a las posibilidades de atención médica (urgente y rápida) en los sistemas hospitalarios con que cuenta el país, la existencia de endocrinólogos pediátricos provinciales, además de la educación sobre diabetes que se brinda a pacientes, familiares, así como a la comunidad donde viven. También a la insistencia del personal de salud que atiende a estos niños, de lograr siempre el mejor control metabólico posible (antes y con énfasis durante la pandemia). Es importante insistir que la diabetes descontrolada (con hiperglucemia) asociada al descontrol metabólico causado por el COVID-19 puede influir de forma negativa en la vida del paciente. Debido a que la afección por COVID-19 puede ocasionar daño a nivel pancreático (células beta) transitorio o permanente, es prudente tener un seguimiento a mediano y largo plazo de estos pacientes.

\section{Conclusiones}

La infección por COVID-19 en poblaciones con Diabetes Mellitus tipo 1 (DM1) se ha incrementado no solo favoreciendo la descompensación metabólica, sino facilitando el debut de esta condición médica. En este estudio, existió incremento de los pacientes al debut (N: 90) de la DM1 durante el primer año de la pandemia $(57.3 \%)$, comparados con los que iniciaron la enfermedad diabética ( $\mathrm{N}$ : 67) en el año previo (2019), también al evaluar la presencia de cetoacidosis/cetosis se constató comparando con el año previo a la pandemia (2019) incremento (58.2 \%) durante el primer año de la pandemia. Se considera que es baja la frecuencia de DM1 y COVID-19 $(0.53 \%)$. No hay señalamientos de fallecidos en la etapa estudiada. Los resultados de esta investigación pueden estar relacionados de forma indirecta con la pandemia, ya sea por stress (individual, socia o ambos), confinamiento, alimentación familiar inadecuada, poca movilidad de la población, etc. o varios de estos aspectos juntos.

Declaración de intereses: los autores declaran que no hay conflictos de interés.

\section{Bibliografía}

1. Acosta J, Pérez M, Rodríguez M, Morales A. COVID-19 en pediatría: aspectos clínicos, epidemiológicos, inmunopatogenia, diagnóstico y tratamiento. Revista Cubana de Pediatría, 2020;92(Supl. especial):e1152.

2. Ministerio de Salud Pública. Informe situación epidemiológica del COVID-19. Cuba; 22 de mayo 2021

3. Noda A, Castro B, López L, Robaina G. Síndrome inflamatorio multisistémico en niños asociado a COVID-19. Revista Cubana de Pediatría, 2020;92(Supl. especial):e1202.
4. Martínez E, Sabatier J. Dengue y COVID-19: semejanzas y diferencias. Revista Cubana de Pediatría, 2020;92(Supl. especial):e1211.

5. López L, et al. Caracterización clínico epidemiológica de 183 niños cubanos con infección por SARS-CoV-2. Revista Cubana de Pediatría, 2020;92(Supl. especial):e1256.

6. Zubkiewicz A, Seifert M, Stępkowski M, NoczyńskaA A. Diagnosis of type 1 diabetes during the SARS-CoV-2 pandemic: Does lockdown affect the incidence and clinical status of patients? Adv Clin Exp Med, 2021;30(2): $127-34$.

7. DiMeglio L, Albanese-O’Neill A, Muñoz C, Maahs D. COVID-19 and Children with Diabetes Updates, Unknowns, and Next Steps: First, Do No Extrapolation. Diabetes Care, 2020;43:2631-4. Available from: https://doi. org/10.2337/dci20-0044

8. Trevisani V, et al. COVID-19 and Type 1 Diabetes: Concerns and Challenges. Acta Biomed, 2020;91(3):1-6. e2020033

9. Kusuma $S$, et al. New onset diabetes, type 1 diabetes and COVID-19. Diabetes \& Metabolic Syndrome: Clinical Research \& Reviews, 2020;14:2211-7

10. Klatman E, etal.COVID-19 and type 1 diabetes: Challenges and actions. Diabetes research and clinical practice, 2020;166(108275):1-4

11. Chao L, Vidmar A, Georgia S. Spike in Diabetic Ketoacidosis Rates in Pediatric Type 2 Diabetes During the COVID-19. Diabetes Care, 2021:44:1-4. Available from: https://doi. org/10.2337/DC20-2733 
12. Ebekozien $\mathrm{O}$, et al. Type 1 Diabetes and COVID-19: Preliminary Findings from a Multicenter Surveillance Study in the U.S. Diabetes Care, 2020;43:e83-e85. Available from: https:// doi.org/10.2337/dc20-1088.

13. Tittel S, et al. Did the COVID-19 Lockdown Affect the Incidence of Pediatric Type 1 Diabetes in Germany? Diabetes Care, 2020;43:e172e173. Available from https://doi.org/10.2337/ dc20-1633.

14. Lawrence $\mathrm{C}$, et al. Increased paediatric presentations of severe diabeticketoacidosis in an Australian tertiary centre during the COVID-19 pandemic. Diabetic Medicine, 2020;00:e14417:1-5.

15. Unsworth R, et al. New-Onset Type 1 Diabetes in Children During COVID-19: Multicenter Regional Findings in the U.K. Diabetes Care, 2020;43:e170-e171. Available from: https:// doi.org/10.2337/dc20-1551

16. d'Annunzio G, et al. Caring for children and adolescents with type 1 diabetes mellitus: Italian Society for Pediatric Endocrinology and Diabetology (ISPED) statements during COVID-19 pandemia. Diabetes research and clinical practice, 2020;168(108372):1-11.

17. Dżygało K, et al. Increased frequency of severe diabetic ketoacidosis at type 1 diabetes onset among children during COVID-19 pandemic lockdown: an observational cohort study. Pediatr Endocrinol Diabetes Metab, 2020;26 (4):167-75. Available from: https://doi.org/10. 5114/pedm.2020.101003

18. González J, Tejado M, Jiménez J. Repercusión del confinamiento por COVID-19 sobre el control glucémico en niños y adolescentes con diabetes mellitus tipo 1. Anales de Pediatría, Available from: https://doi.org/10.1016/j. anpedi.2020.12.021
19. Odeh R, Gharaibeh L, Dahera A, Kussad S, Alassaf A. Caring for a child with type 1 diabetes during COVID-19 lockdown in a developing country: Challenges and parents' perspectives on the use of telemedicine. Diabetes research and clinical practice, 2020;168(108393):1-7.

20. Sánchez M, González J, Tejado M, Jiménez J. Repercusión del confinamiento por COVID-19 sobre el control glucémico en niños y adolescentes con diabetes mellitus tipo 1, Anales de Pediatría. Available from: https://doi.org/10.1016/j. anpedi.2020.12.021

21. Li G, Fan Y, Lai Y, Han T, Li Z, Zhou P, et al Coronavirus infections and immune responses. J Med Virol [Online]. 2020;92:424-32. Available from: https://doi.org/10.1002/jmv.25685

22. Li F. Structure, function, and evolution of coronavirus spike proteins. Annu Rev Virol [Online]. 2016;3:237-61. Available from: https://doi.org/ 10.1146/annurev-virology-110615-042301

23. Muniyappa R, Gubbi S. COVID-19 pandemic, corona viruses, and diabetes mellitus. Am J Physiol Endocrinol Metab [Online]. 2020. Available from: https://doi.org/10.1152/ajpendo. 00124.2020

24. Hoffmann M, Kleine-Weber H, Schroeder S, Kruger N, Herrler T, Erichsen S, et al. SARS-CoV-2 cell entry depends on ACE2 and TMPRSS2 and is blocked by a clinically proven protease inhibitor. Cell [Online]. 2020. Available from: https://doi.org/10.1016/j.cell. 2020.02.052.

25. Raj VS, Mou H, Smits SL, Dekkers DH, Muller MA, Dijkman R, et al. Dipeptidylpeptidase 4 is a functional receptor for the emerging human coronavirus-EMC. Nature [Online]. 2013;495:251-4. Available from: https://doi. org/10.1038/nature12005
26. Lin L, Lu L, Cao W, Li T. Hypothesis for potential pathogenesis of SARS-CoV-2 infection-are view of immune changes in patients with viral pneumonia. Emerg Microbes Infect [Online]. 2020;9:727-32. Available from: https://doi.org/ 10.1080/22221751.2020.1746199
27. Zhao J, Yuan Q, Wang H, Liu W, Liao X, Su $\mathrm{Y}$, et al. Antibody responses to SARS-CoV-2 in patients of novel coronavirus disease 2019. Clin Infect Dis [Online]. 2020. Available from: https://doi.org/ 10.1093/cid/ciaa344 